\title{
Health promotion and healthy eating habits. Exploratory study in primary education.
}

\author{
Encarnación Pedrero-García ${ }^{1, *}$, Olga Moreno-Fernández ${ }^{2}$, Pilar Moreno-Crespo ${ }^{2}$, and \\ Carmen Solís-Espallargas ${ }^{2}$ \\ ${ }^{1}$ Univerisidad Pablo de Olavide, Crta. de Utrera, Km. 1, Sevilla 41013, Spain \\ ${ }^{2}$ Universidad de Sevilla,Pirotecnica, 19, Sevilla 41013, Spain
}

\begin{abstract}
This paper focuses on the importance of health education in Primary Education and the role of schools in the acquisition of healthy habits, especially in nutrition. The aim of this study is to provide an approximation of what students in the 6th year of Primary Education (11-12 years old) understand by healthy habits related to nutrition. A questionnaire of six openended questions was carried out, with a total sample of 78 children. It should be noted that a large sample confuses healthy eating with good health. We therefore consider the need to favour greater health education in school classrooms, as this would help them to differentiate balanced diets from those that are not and, therefore, to prepare healthy menus appropriately.
\end{abstract}

\section{Introduction}

This research focuses on the importance of health education in Primary Education and on the role of the school in the acquisition of healthy habits, especially in nutrition. The possibility of intervening educationally makes it necessary to analyze what knowledge primary school children have about the different concepts and habits related to nutrition. In the ages of 6 to 12 years, children are in constant growth and development, and begin to develop eating patterns, being important to maintain a balance in their nutrients controlling their carbohydrates, proteins and fats, with the aim of contributing to good physical development, psychic and prevent diseases related to poor nutrition such as cavities, hypertension or obesity [1]. Specifically, obesity is one of the most serious public health problems of the 21 st century according to the World Health Organization [2]. The problem is global and progressively affects many low- and middle-income countries, especially in the urban environment. Prevalence has increased at an alarming rate.

In recent years, health education has become vitally important in education. This development is supported by institutions such as World Health Organization, UNESCO, UNICEF, the Council of Europe, the European Commission and the Organisation for Economic Co-operation and Development (OECD). The educational context has consolidated itself as one of the most suitable places to implement Health Education programs, being therefore a relevant context to promote the long-term adoption of healthy

\footnotetext{
*Corresponding author: epedgar@upo.es
} 
lifestyles. For the World Health Organization [3] "a health promoting school is defined as one that constantly strengthens the characteristics that make it a healthy environment in which to live, learn and work. Therefore, when we refer to health-promoting schools, they must meet a number of characteristics. Castillo (1998:43) [4] points out the following:

- It considers health education through all aspects of school life and its relations with the community and not only in class activities.

- It is based on an integral health model that includes interactions between physical, mental, social and environmental aspects.

- It focuses on the active participation of students through a wide variety of methods that contribute to the development of skills.

- Recognizes that student health is subject to multiple influences and takes into account pre-existing beliefs, values, and attitudes.

- It recognizes that there are skills and processes that form the basis of all health improvement and include them as objectives in the school curriculum.

- It tends to develop in the student a positive self-image, and to increase their capacity for self-control, as basic elements of the promotion of good health.

- It recognises the importance of the physical environment and its physiological and effective effects on students and school staff.

- Understands the promotion of health in the school with the welfare of school staff and recognizes the role of example that plays with the child.

- It considers the parents of the pupils as an indispensable support for the school in the promotion of health.

- Involves school medical services to participate in the educational program and help students make better use of the resources they can offer.

In Spain, health education is included in the curriculum as a cross-cutting theme, that is, as a teaching that does not belong to a particular area of curriculum development, but must be present in all areas. Through the different regional norms of curriculum development of the stages of infant, primary, secondary, high school education, adult education is based on the need for health education. In Andalusia, the context in which this research focuses on Primary Education, a specific Plan for the promotion of healthy habits has been implemented. Therefore, health education must be implemented in the school at the level of objectives, contents, activities, methodology and evaluation. In short, it must be formally considered within the status provided by the educational project of the centers [5].

\section{Method}

The aim of this study is to provide an approximation of what students in the 6th year of Primary Education (11-12 years old) understand by healthy habits related to nutrition. We had a sample of 78 Primary Education students enrolled in schools in the province of Seville (Spain). In order to carry out the exploratory study, we have used an open-ended questionnaire in which six items related to food and healthy habits are collected, as mentioned below:

1. Could you define in your own words what a healthy diet or a good diet is?

2. Of the foods presented to you below, could you circle those that you consider healthy?

3. Could you indicate with arrows in which part of the food pyramid the foods proposed to you go?

4. What would a healthy menu be for you? Make your own menu.

5. Could you put a sad face on the less healthy foods and a happy face on the healthier ones?

6. Would you know how to classify the following foods? 


\section{Results}

$35.7 \%$ confuse healthy eating with good health, while $57.1 \%$ do not understand healthy eating as separate from physical exercise, pointing out the importance of a good diet accompanied by some form of sport. Only $7.2 \%$ of respondents did not answer on the basis of what was asked (Fig. 1).

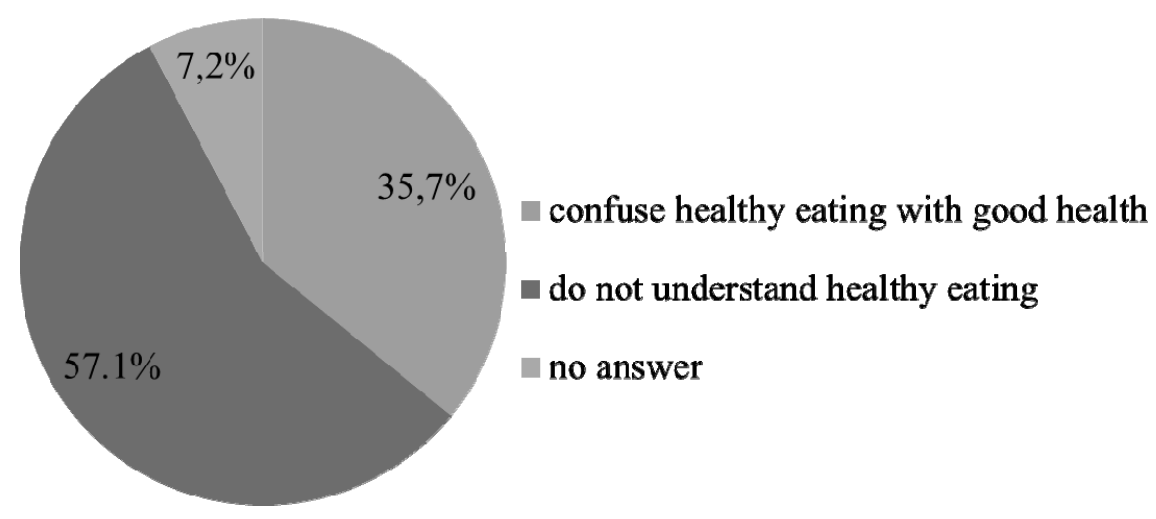

Fig. 1. Healthy diet/good health.

As for the design of a healthy menu, $92.8 \%$ are able to select healthy foods when designing a menu; however, they are not able to elaborate a complete and varied menu, as well as having difficulty distinguishing between first and second course (Fig. 2).

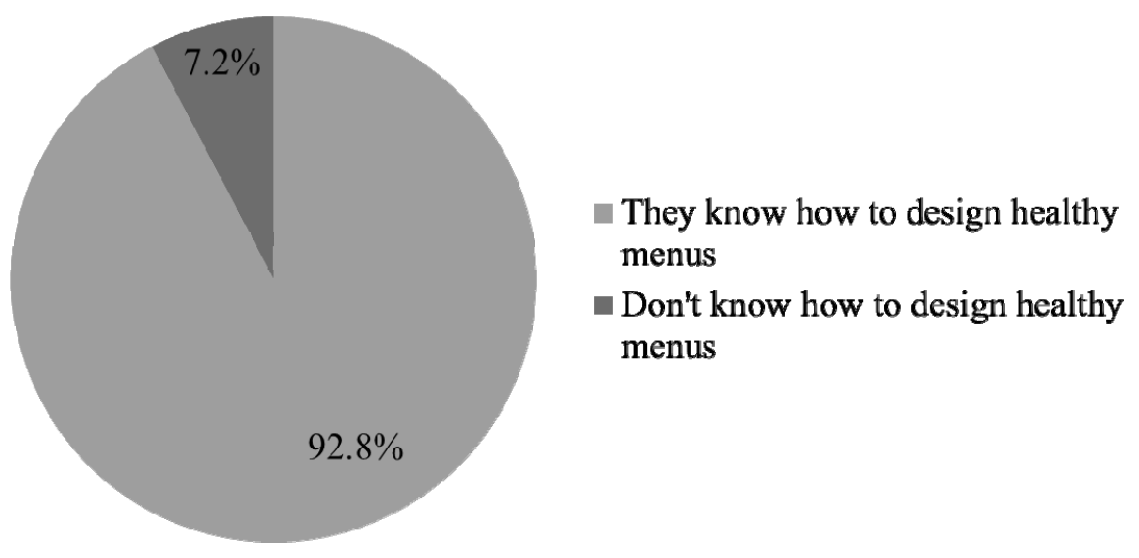

Fig. 2. Design of a healthy menu.

As for the food pyramid, we found different kinds of answers:

a) $24.8 \%$ of students confuse the cusp with the base and put the food on the contrary.

b) $35.9 \%$ do not know very well where to place each food.

c) $42.3 \%$ place each food in its place. 


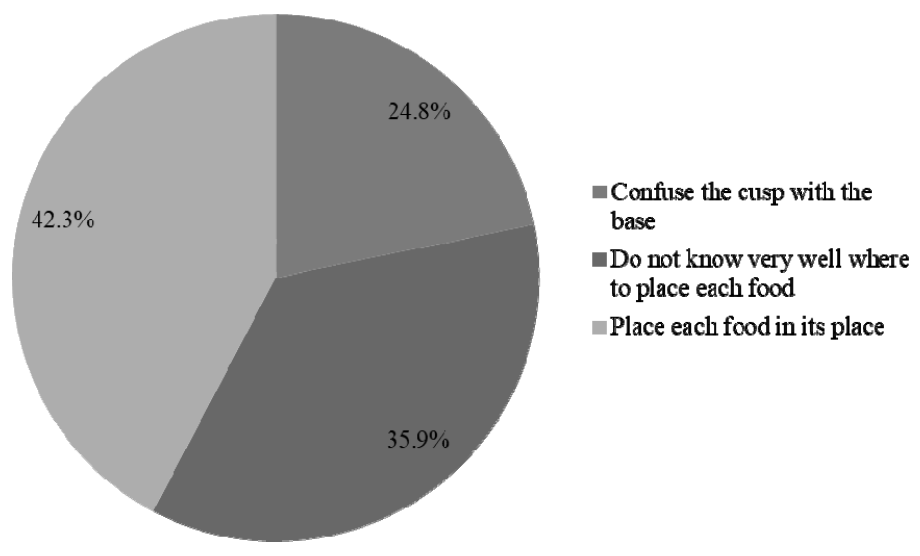

Fig. 3. The food pyramid.

Observing (Fig. 3) the results obtained, we consider it necessary to work the food pyramid in the classroom, making it clear which foods we should consume more or less frequently, as well as their correspondence with the cusp or the base.

Finally, it should be noted that when it comes to identifying the types of food, $100 \%$ of the sample classifies fruit well and $85 \%$ identifies vegetables without any problem. However, $35.7 \%$ are not able to identify cereals and $42.8 \%$ are able to identify legumes (Fig. 4).

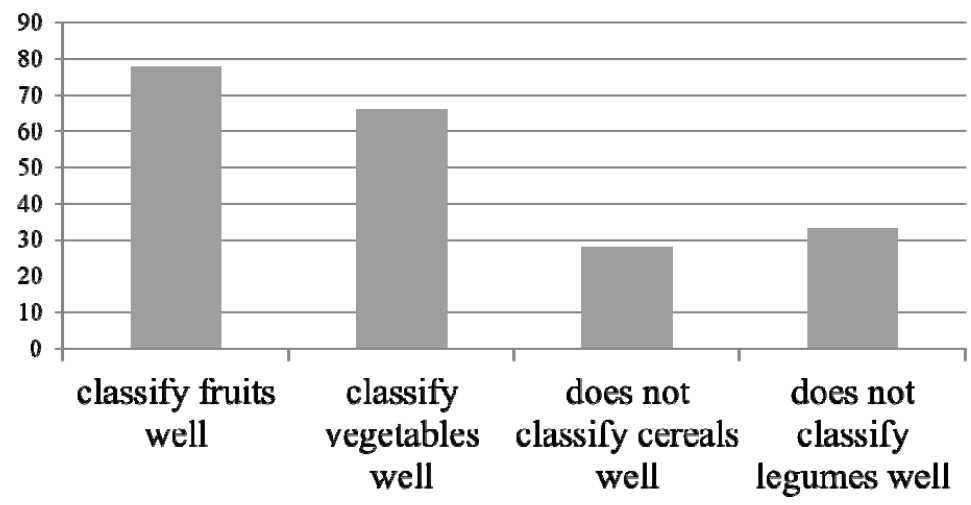

Fig. 4. Types of food.

\section{Conclusions}

Health education can be developed in all areas in which the person lives. In primary education, special attention must be paid to the family, the school and the community. We consider it relevant to work on this content in the classroom, as it would help them to differentiate balanced diets from those that are not and, therefore, to prepare healthy menus appropriately. Health Education is an area of great relevance in the acquisition of healthy habits in the school environment, so it is necessary to develop more educational programs for this purpose. 
We agree with Rosales (2014:301) [6] "that far from thinking of an independent intervention, in the field of health education as in other educational issues, the need for coordination between the agents involved is highlighted. Far from thinking of an independent intervention, in the field of health education as in other educational issues, the need for coordination between the actors involved is highlighted".

\section{References}

1. R. García, Revista Digital Innovación y Experiencias Educativas, 29 (2010)

2. World Health Organization, UNESCO strategy on education for health and well-being: contributing to the Sustainable Development Goals (2016)

3. World Health Organization, Health Promotion. Glossary (1998)

4. F. Castillo, La escuela promotora de salud [The health promoting school] (1998).

5. L. S. Leger, L. Kolbe, A. Lee, D. McCall, I. Young, School health promotion: achievements, challenges and priorities (2007).

6. C. Rosales, Innovación Educativa 24, 289-303 (2014). 